\title{
Alterations of the tumor microenvironment in glioblastoma following radiation and temozolomide with or without bevacizumab
}

\author{
Ryota Tamura ${ }^{1}$, Toshihide Tanaka ${ }^{2}$, Yukina Morimoto ${ }^{1}$, Yuki Kuranari ${ }^{1}$, Yohei Yamamoto ${ }^{2}$, Jun Takei ${ }^{2}$, \\ Yuichi Murayama ${ }^{3}$, Kazunari Yoshida ${ }^{1}$, Hikaru Sasaki ${ }^{1}$ \\ ${ }^{1}$ Department of Neurosurgery, Keio University School of Medicine, Shinjuku-ku, Tokyo, Japan; ${ }^{2}$ Department of Neurosurgery, Jikei University \\ Kashiwa Hospital, Kashiwa-shi, Chiba, Japan; ${ }^{3}$ Department of Neurosurgery, Jikei University Hospital, Minato-ku, Tokyo, Japan \\ Contributions: (I) Conception and design: R Tamura, H Sasaki, T Tanaka; (II) Administrative support: T Tanaka, H Sasaki; (III) Provision of study \\ materials or patients: T Tanaka, H Sasaki, Y Murayama, K Yoshida; (IV) Collection and assembly of data: R Tamura, T Tanaka, Y Morimoto, Y \\ Kuranari, H Sasaki; (V) Data analysis and interpretation: R Tamura, Y Morimoto, Y Kuranari, H Sasaki, T Tanaka; (VI) Manuscript writing: All \\ authors; (VII) Final approval of manuscript: All authors. \\ Correspondence to: Toshihide Tanaka, MD, PhD. Department of Neurosurgery, Jikei University Kashiwa Hospital, 163-1 Kashiwashita, Kashiwa-shi, \\ Chiba, 277-8567, Japan. Email: ttanaka@jikei.ac.jp.
}

\begin{abstract}
Background: The immunosuppressive tumor microenvironment (TME) contributes to the tumor progression and treatment failure. Our previous study demonstrated alterations in the TME during bevacizumab (Bev) therapy in human glioblastoma (GB) specimens obtained from patients who underwent surgical resection. Continuous Bev administration downregulates the expression of programmed cell death-1 (PD-1)/programmed cell death ligand-1 (PD-L1), suppresses the infiltration of tumor associated macrophages (TAMs) and regulatory $\mathrm{T}$ cells (Tregs), and increases cytotoxic $\mathrm{T}$ lymphocytes (CTLs) infiltration. However, one may argue that these immunosupportive effects might also be induced by radiation therapy (RT) or temozolomide (TMZ), and they cannot necessarily be attributed to Bev alone.

Methods: In the present study, changes in the molecules relevant to the TME were analyzed by immunohistochemistry using paired pre- and post-treatment samples of malignant glioma specimens from 15 patients who received RT and TMZ therapy without Bev.

Results: The expression levels of CD34, vascular endothelial growth factor (VEGF)-A, VEGF receptor 2 (VEGFR2), HIF-1 $\alpha$, CA9, nestin, CD4, CD8, CD163, PD-1, and PD-L1 were not significantly changed after the treatment with RT and TMZ. However, VEGFR1 expression and the number of Foxp3-positive cells tended to be upregulated and increased after the treatment $(\mathrm{P}=0.058, \mathrm{P}=0.082$, respectively).

Conclusions: This was the first study to show the alterations of TME following RT and TMZ therapy using paired pre- and post-treatment malignant glioma samples. Long-term treatment of RT and TMZ might worsen immunosuppressive TME in malignant gliomas.
\end{abstract}

Keywords: Temozolomide; radiation; glioblastoma (GB); regulatory T cell; tumor-associated macrophage

Submitted Dec 17, 2019. Accepted for publication Feb 19, 2020.

doi: $10.21037 / \mathrm{atm} .2020 .03 .11$

View this article at: http://dx.doi.org/10.21037/atm.2020.03.11

\section{Introduction}

The tumor microenvironment (TME) exerts an immunosuppressive effect and plays critical roles in tumor growth, angiogenesis, and metastasis (1). The lack of anti- tumor immune response caused by an immunosuppressive TME is associated with the refractoriness of treatment for glioblastomas (GBs) (2). The vascular endothelial growth factor (VEGF) is induced by hypoxia through a hypoxia- 

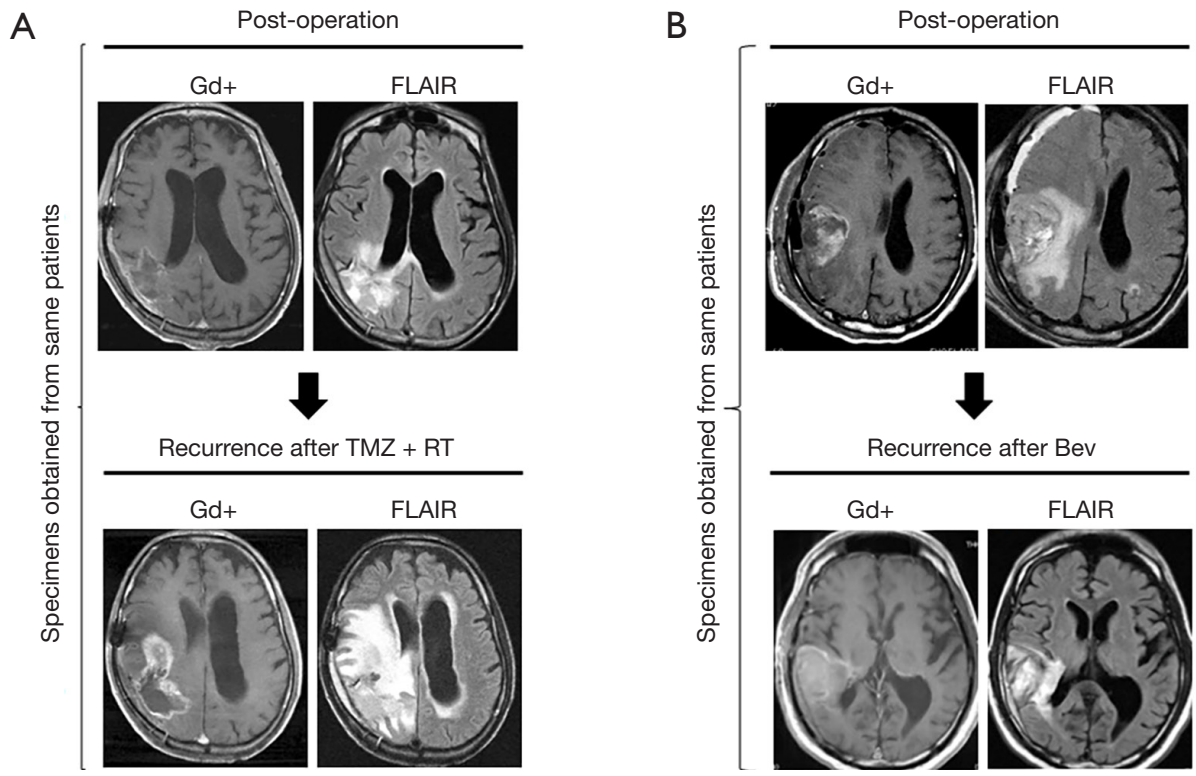

Figure 1 Design of the present study. In the present study, alterations in molecules that are relevant to the TME are analyzed using paired pre- and post-RT and TMZ specimens from 15 patients with malignant glioma (A). These results are compared with previously reported results using paired pre- and post-Bev therapy (B). T1-weighted images with contrast enhancement and FLAIR images acquired after operation and at the recurrent stage after the Stupp regimen (RT and TMZ) or Bev therapy are shown. Bev, bevacizumab; Gd+, T1weighted images with contrast enhancement; FLAIR, FLuid-Attenuated Inversion Recovery; RT, radiotherapy; TMZ, temozolomide.

inducible factor 1 alpha (HIF-1 $\alpha)$-depending pathway, which contributes to immune suppression in the TME (3). Therefore, agents that target VEGF [bevacizumab (Bev)] or the VEGF receptor (VEGFR; sunitinib and sorafenib) are expected to induce not only anti-angiogenic effects, but also immunosupportive effects (4).

We have previously analyzed the alterations of the TME following Bev therapy using actual human GB specimens. Bev downregulated the expression of programmed cell death-1 (PD-1)/programmed cell death ligand-1 (PD$\mathrm{L} 1)$, suppressed the infiltration of regulatory $\mathrm{T}$ cells (Tregs) and tumor-associated macrophages (TAMs), and increased cytotoxic T lymphocytes (CTLs) infiltration (5). Importantly, this immunosupportive condition was sustained during continuous Bev usage. However, one may argue that these immunosupportive effects may also be induced by the standard treatment protocol using Stupp regimen [radiation therapy (RT) concomitant with temozolomide (TMZ) and adjuvant TMZ] (6), and they cannot necessarily be attributed to Bev.

Therefore, in the present study, alterations in the molecules that are relevant to the TME were analyzed using paired pre- and post-RT and TMZ specimens from 15 patients with malignant glioma who did not receive Bev therapy, to confirm the differential actions between Bev and Stupp protocol.

\section{Methods}

This study was approved by the Institutional Review Board of our institutes (Reference number: 20050002 and 26-334). Written consent was obtained from all patients.

\section{Tissue specimens}

In the present study, we analyzed paired pre- and post-RT and TMZ specimens from 15 patients with malignant glioma (30 tumor tissues) (Figure 1A). All tissue specimens were obtained by surgical operation at Keio University School of Medicine and Jikei University Kashiwa Hospital, Japan between January 2009 and December 2018. The analyses were performed at Keio University School of Medicine. The present results were compared with those of our previous studies regarding Bev administration (5,7,8) (Figure 1B).

\section{Immunobistochemical analyses}

Immunohistochemical analyses were performed, as 
previously described $(5,7,8)$. The following antibody were used: anti-VEGF-A (1:200, JH121, Merck Millipore), anti-VEGF receptor (VEGFR)1 (1:200, AF321, R\&D SYSTEMS), anti-VEGFR2 (1:600, 55B11, Cell Signaling Technology), anti-CD34 (1:100, F1604, Nichirei Biosciences Inc.), anti-HIF-1 $\alpha$ (1:100, H-206, Santa Cruz Biotechnology), anti-carbonic anhydrase 9 (CA9) (1:50, H-120, Santa Cruz Biotechnology), anti-nestin (1:100, 10C2, Chemicon), anti-PD-1 (1:50, NAT105, Abcam), anti-PD-L1 (1:500, 28-8, Abcam), anti-CD4 (1:200, 1F6, Nichirei Bioscience Inc.), anti-CD8 (1:100, 144B, Abcam), anti-Foxp3 (1:100, ab54501, Abcam), and anti-CD163 (1:100, ab87099, Abcam). The status of the TME after Bev administration in our previous studies (5) was also highlighted using the original figures (Figure 1B). The results of the immunohistochemical analysis were evaluated, as previously described $(5,7,8)$ (noted in Table 1 ).

\section{Statistical analyses}

Paired $t$-test was used for the paired comparison of the markers described above (CD34, nestin, CD4, CD8, Foxp3, CD163, and PD-1). Chi-squared test was applied to compare the scores of VEGF-A, VEGFR1/R2, HIF-1 $\alpha$, CA9 and PD-L1 expressions. Student's $t$-test was used for the comparison of percent change in markers from baseline in the patients who received $\geq 10$ courses of TMZ $v s$. those who did not. All statistical analyses were performed using IBM SPSS statistics. P value less than 0.05 was considered to be statistically significant.

\section{Results}

\section{Patients characteristics}

The general information of the patients, as well as their location and histology results, are summarized in Table 2. Fourteen cases were wild type for isocitrate dehydrogenase (IDH)1-R132H, whereas O6-methylguanine DNA methyltransferase (MGMT) promoter methylation was observed in 8 cases. The median number of courses of TMZ was five (mean, 6.6 courses). The median overall survival was 603 days (72-1,142 days) (Table 2).

\section{Histological and vascular changes}

The recurrent tumors following RT and TMZ showed proliferation of astrocytes with nuclear anaplasia, and the form of tumor cells was essentially similar to that of the previous surgery (data not shown). Palisading necrosis and microvascular proliferation were also observed. Microvessel density (MVD) was not decreased in the tumors after RT and TMZ compared with that before the treatment (pre, 32.9 \pm 12.76/5HPF; post, 33.9 $\pm 11.29 / 5 \mathrm{HPF}, \mathrm{P}=0.41$ ) (Figure 2A,B) (Table 1).

\section{VEGF-A/VEGFRs expressions}

VEGF-A staining was diffuse intense staining $(++)$ in $8 / 15$ and $10 / 15$ tumors before and after RT and TMZ, respectively $(\mathrm{P}=0.46)$ (Figure $2 A, B)$. VEGFR1 staining was observed in endothelial cells and tumor cells in $3 / 15$ and $8 / 15$ tumors before and after RT and TMZ, respectively $(\mathrm{P}=0.058)$ (Figure $2 A, B$ ). VEGFR2 staining was detected in endothelial cells and tumor cells in 2/15 and 1/15 tumors before and after RT and TMZ, respectively $(\mathrm{P}=0.54)$ (Figure $2 A, B)$.

\section{Expression of hypoxic marker}

Fourteen tumors before the RT and TMZ exhibited positive $(+$ and ++$)$ staining for HIF-1 $\alpha$, while all tumors after the treatment showed positive $(+$ and ++$)$ staining for the marker $(\mathrm{P}=0.46)$ (Figure $2 A, B)$. The CA9 staining was universal or occasional in $9 / 15$ and $10 / 15$ tumors before and after RT and TMZ, respectively $(\mathrm{P}=0.54)$ (Figure $2 A, B)$.

\section{Nestin expression}

Although numerous nestin-positive tumor cells were observed in all pre- and post- tumors treated with RT and TMZ, there was no difference of nestin-positive cell ratio of tumors before $v s$. after the RT and TMZ (pre, $40.8 \% \pm 22.31 \%$; post, $37.73 \% \pm 24.73 \%, \mathrm{P}=0.36$ ) (Figure 2A,B).

\section{PD-1/PD-L1 expressions}

There was no difference in the number of PD-1 (+) cells between tumors before $v s$. after the RT+TMZ (pre, 3.47 $\pm 2.89 / 5 \mathrm{HPF}$; post, $3.73 \pm 2.34 / 5 \mathrm{HPF}, \mathrm{P}=0.39$ ) (Figure $3 A, B)$. Positive $(+++)$ staining for PD-L1 was observed in four out of 15 tumors before and in three out of 15 tumors after the RT and TMZ $(\mathrm{P}=0.66)$ (Figure $3 A, B)$.

\section{CD4 and CD8 expressions}

There was no differences in the number of CD4 (+) and 
Table 1 Results of immunohistochemical analyses

\begin{tabular}{|c|c|c|c|c|c|c|c|c|c|c|c|c|c|}
\hline Case & VEGF-A & VEGFR1 & VEGFR2 & CD34 & Nestin \% & $\mathrm{HIF}-1 \alpha$ & CA9 & $\begin{array}{l}\text { PD-L1 } \\
\text { (score) }\end{array}$ & $\begin{array}{c}\text { PD-1 } \\
\text { (/5HPF) }\end{array}$ & $\begin{array}{c}\text { CD4 } \\
(/ 5 \mathrm{HPF})\end{array}$ & $\begin{array}{c}\text { CD8 } \\
\text { (/5HPF) }\end{array}$ & $\begin{array}{l}\text { Foxp3 } \\
\text { (/5HPF) }\end{array}$ & $\begin{array}{l}\text { CD163 } \\
\text { (/5HPF) }\end{array}$ \\
\hline 1 pre & ++ & ++ & + & 64 & 80 & + & - & 2 & 12 & 24 & 14 & 4 & 33 \\
\hline 2 pre & + & + & + & 34 & 60 & ++ & + & 3 & 5 & 12 & 10 & 1 & 42 \\
\hline 2 post & + & ++ & + & 48 & 15 & + & - & 2 & 8 & 8 & 5 & 3 & 38 \\
\hline 3 post & ++ & ++ & + & 36 & 5 & \pm & - & 1 & 1 & 2 & 3 & 2 & 12 \\
\hline 4 pre & ++ & ++ & + & 26 & 25 & \pm & + & 3 & 1 & 2 & 2 & 1 & 22 \\
\hline 4 post & ++ & ++ & + & 52 & 30 & + & + & 3 & 2 & 12 & 2 & 1 & 46 \\
\hline 5 pre & ++ & ++ & + & 21 & 35 & + & + & 3 & 0 & 2 & 3 & 1 & 16 \\
\hline 7 pre & ++ & + & + & 32 & 43 & ++ & + & 1 & 2 & 2 & 12 & 1 & 64 \\
\hline 7 post & ++ & ++ & + & 30 & 31 & + & + & 1 & 7 & 3 & 2 & 4 & 26 \\
\hline 8 pre & ++ & + & + & 19 & 41 & + & + & 1 & 3 & 4 & 3 & 4 & 11 \\
\hline 8 post & ++ & + & + & 24 & 31 & + & ++ & 1 & 1 & 3 & 2 & 8 & 52 \\
\hline 9 pre & + & + & ++ & 26 & 21 & ++ & - & 1 & 3 & 7 & 6 & 2 & 8 \\
\hline 9 post & + & + & ++ & 18 & 28 & ++ & - & 1 & 6 & 10 & 18 & 1 & 48 \\
\hline 10 pre & ++ & + & + & 42 & 31 & ++ & ++ & 1 & 3 & 15 & 14 & 4 & 36 \\
\hline 13 post & ++ & + & + & 30 & 14 & ++ & + & 1 & 2 & 10 & 6 & 5 & 44 \\
\hline 14 pre & + & + & + & 40 & 42 & ++ & + & 1 & 1 & 3 & 30 & 2 & 46 \\
\hline 14 post & ++ & ++ & + & 38 & 64 & ++ & + & 2 & 4 & 1 & 11 & 2 & 12 \\
\hline 15 pre & ++ & + & + & 18 & 64 & ++ & + & 2 & 3 & 3 & 4 & 4 & 50 \\
\hline 15 post & + & + & + & 21 & 58 & + & + & 2 & 2 & 7 & 14 & 5 & 64 \\
\hline
\end{tabular}

For the assessment of microvessel density (MVD), the tissue sections were screened in low-power fields ( $x 4)$, and the three most vascularized regions (hot spots) were selected for each region. The counting of microvessels was performed on these regions at high-power fields $(\times 20$, $0.95 \mathrm{~mm}^{2}$ ). VEGF-A expression in the tumor cytoplasm or stroma was assessed as follows: ++, diffuse intense staining; +, diffuse faint staining; -, negative staining. The expressions of VEGFR1 and VEGFR2 on endothelial or tumor cell membrane/cytoplasm were assessed: ++ , staining in both vascular endothelial cells and tumor cells; +, staining only in vascular endothelial cells; -, negative staining. Expression of $\mathrm{HIF}-1 \alpha$ was assessed as: ++ , expression in $>10 \%$ of tumor cells; + , expression in $\leq 10 \%$ of tumor cells; - , negative staining. Expression of CA9 was assessed as: ++ , universal strong expression around necrotic regions; +, occasional expression (typically around necrotic regions); -, negative staining. Expression of nestin was assessed as a positive cell ratio analyzed in more than 1,000 tumor cells from more than three areas, showing the representative appearance of each tumor. For the assessment of PD-1, CD3, CD4, CD8, Foxp3, and CD163, the stained sections were screened at low-power fields $(\times 4)$, and 5 hot spots were selected. Then, the number of positive cells in these areas was counted in high-power fields $\left(\times 40,0.47 \mathrm{~mm}^{2}\right)$. The expression of PD-L1 was scored as a percentage of tumor cells expressing PD-L1, as described previously. PD-1, programmed cell death-1; PD-L1, programmed cell death ligand-1; post, post-Stupp regimen tumors; pre, preStupp regimen initial tumors; VEGF, vascular endothelial growth factor; VEGFR, vascular endothelial growth factor receptor. 
Table 2 Patients' characteristics

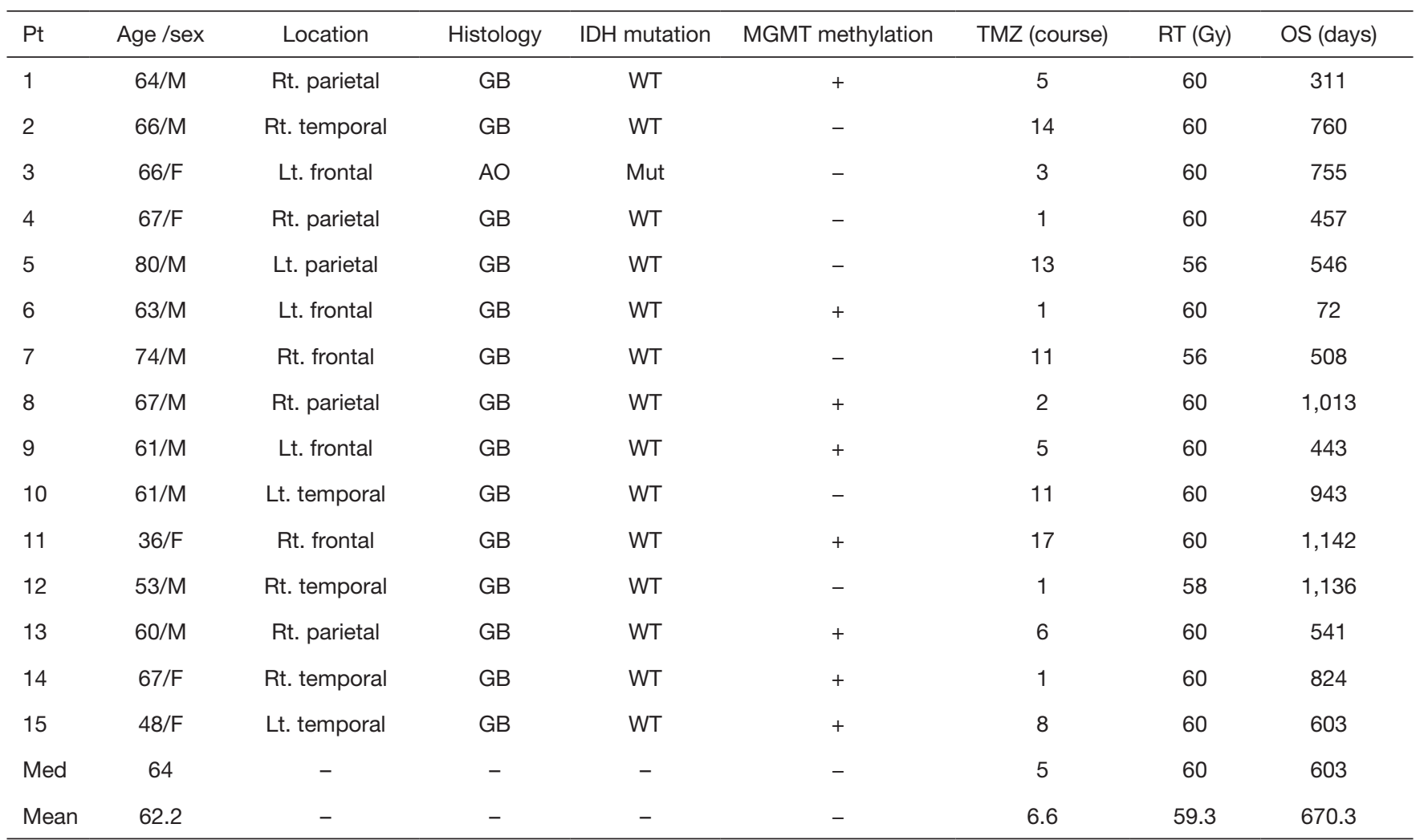

$\mathrm{AO}$, anaplastic oligodendroglioma; F, female; GB, glioblastoma; IDH, isocitrate dehydrogenase; Lt, left; M, male; Med, median; MGMT, O6methylguanine DNA methyltransferase; Mut, mutant; N/A, not available; OS, overall survival; post, post-Stupp regimen tumors; pre, preStupp regimen initial tumors; Pt, patient; Rt, right; RT, radiotherapy; TMZ, temozolomide; WT, wild type.

CD8 (+) cells in tumors before $v s$. after the RT and TMZ (CD4: pre, $6.93 \pm 6.70 / 5 \mathrm{HPF}$; post, $7.47 \pm 6.08 / 5 \mathrm{HPF}$, $\mathrm{P}=0.41$; CD8: pre, $9.33 \pm 6.95 / 5 \mathrm{HPF}$; post, $8.07 \pm 5.19 / 5 \mathrm{HPF}$, $\mathrm{P}=0.29$ ) (Figure $3 A, B)$.

\section{Foxp 3 and CD163 expressions}

The number of Foxp3 (+) cells tended to be increased in tumors after RT and TMZ compared with that observed before the treatment (pre, $2.53 \pm 1.55 / 5 \mathrm{HPF}$; post, $3.6 \pm 2.41 / 5 \mathrm{HPF}, \mathrm{P}=0.082$ ) (Figure $3 A, B)$. There was no difference in the number of CD163 (+) cells in tumors before $v s$. after the RT and TMZ (pre, $31.0 \pm 16.86 / 5 \mathrm{HPF}$; post, $35.33 \pm 18.42 / 5 \mathrm{HPF}, \mathrm{P}=0.25$ ) (Figure $3 A, B$ ).

\section{Association between the number of TMZ courses and the TME}

The percent change from the baseline (pre-RT and TMZ therapy) between the patients who received $\geq 10$ courses $(n=5)$ or $\leq 9$ courses $(n=10)$ of TMZ was evaluated. The patients who received $\geq 10$ courses of TMZ showed an increase in the number of Foxp3 + cells and a decrease in the number of CD163+ cells in tumors after treatment compared with the patients who received $\leq 9$ courses of TMZ (Foxp3, P=0.071; CD163, $\mathrm{P}=0.017$ ) (Figure 4). Tumor oxygenation, stemness, expressions of immune checkpoint molecules, and CTL infiltration did not vary significantly according to the number of TMZ courses.

Case 3 had a diagnosis of the initial and recurrent tumors of anaplastic oligodendroglioma and GB, respectively; however, its exclusion from the statistical analyses did not alter the results.

\section{Highlights of the changes in TME after Bev administration}

Our previous study reported the overall status of the TME, including tumor oxygenation and stemness, in surgical 
A

A Pre-TMZ \& RT
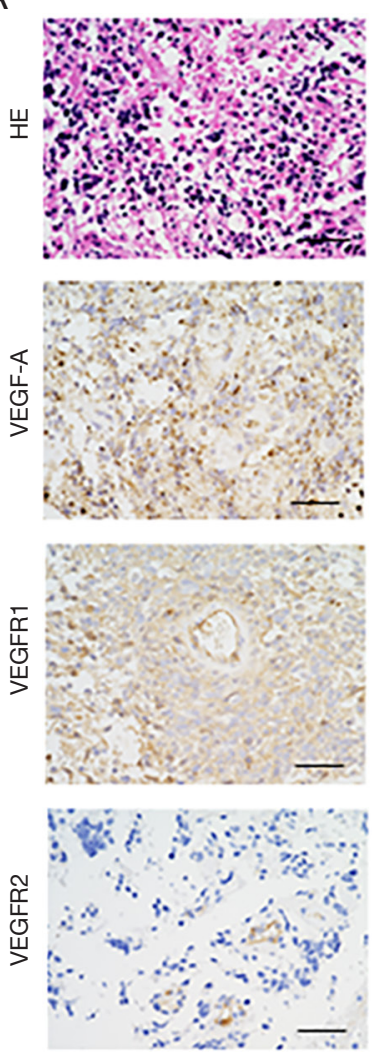

Post-TMZ \& RT
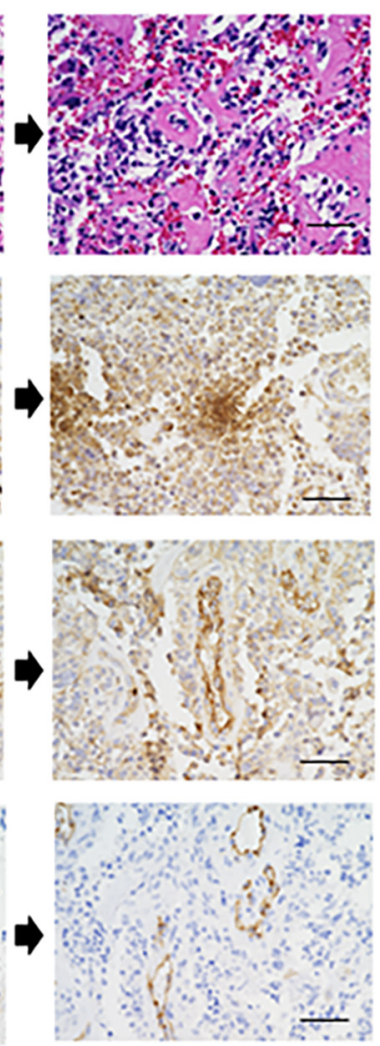
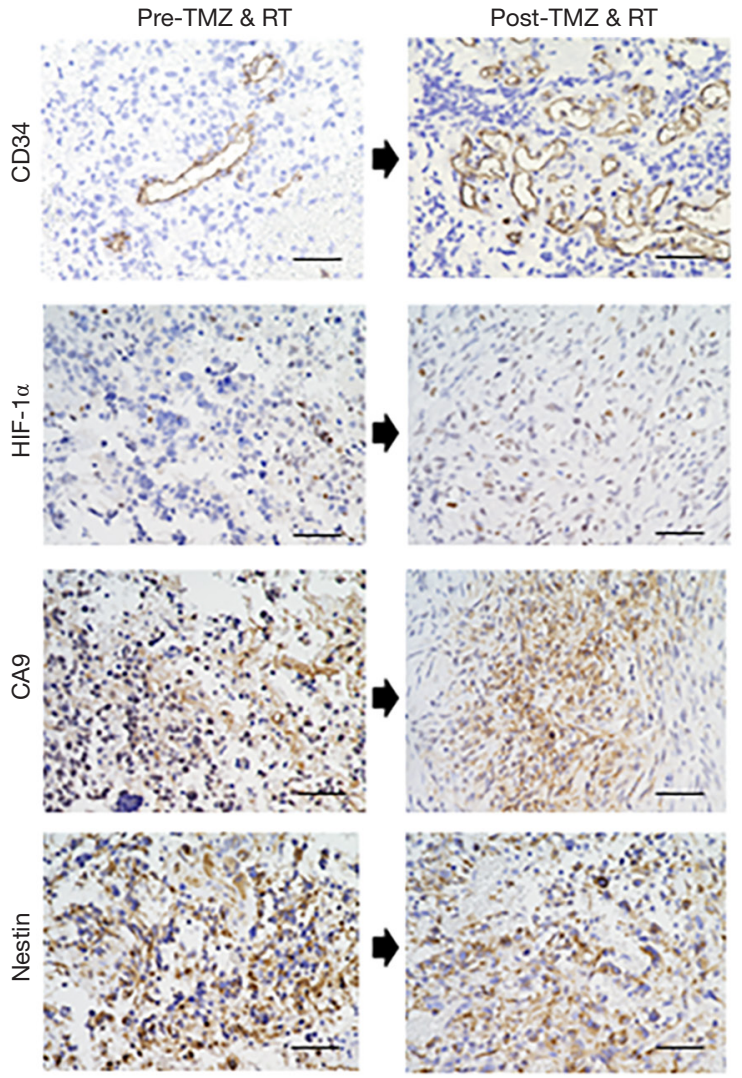

B
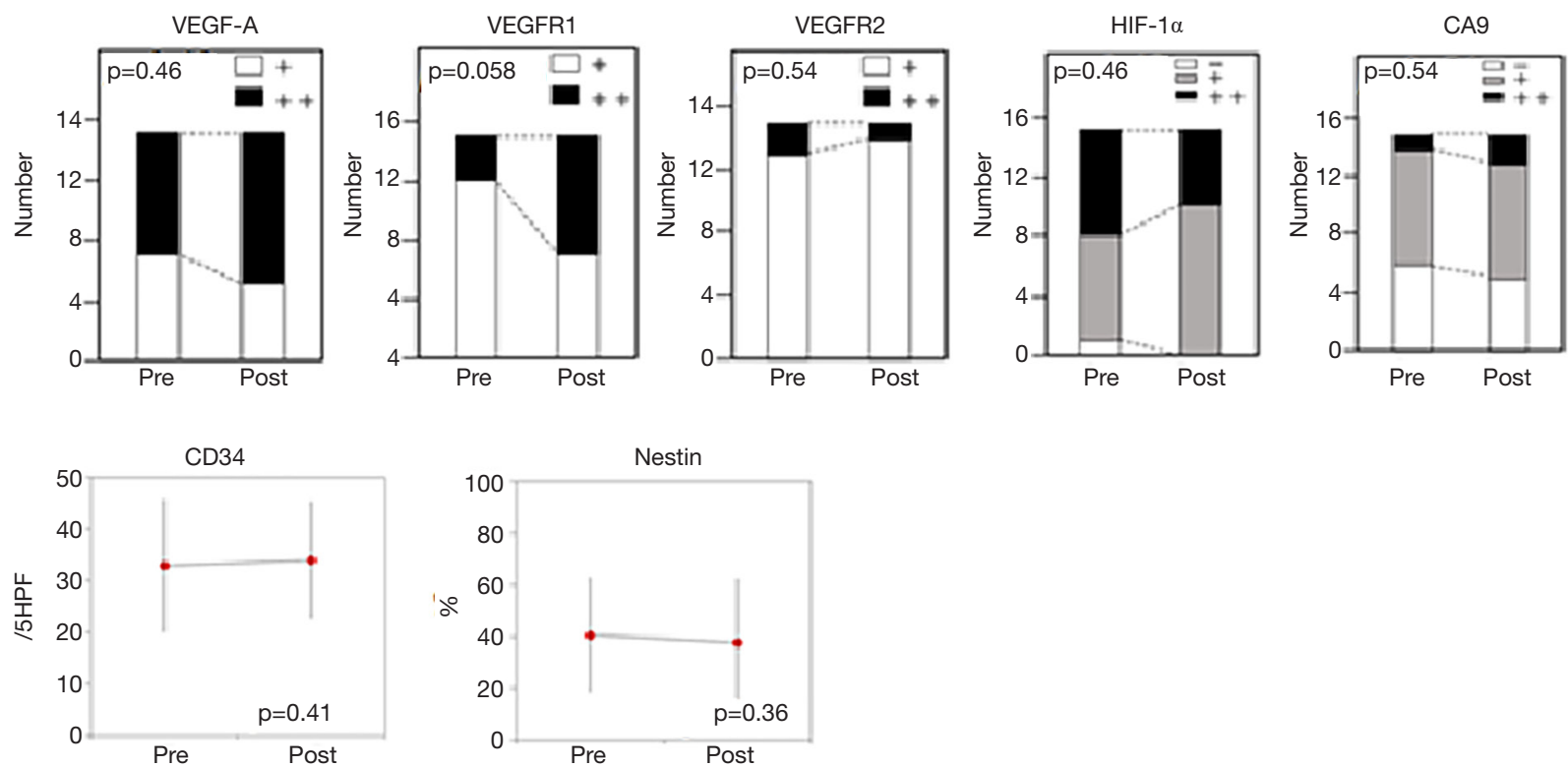

Figure 2 Results of immunohistochemical analyses of angiogenesis, hypoxia, and stemness. Photomicrographs (A) and the results of statistical analyses (B) are shown. Immunohistochemical analyses demonstrate that the expression levels of VEGF-A/VEGFR2, CD34, HIF$1 \alpha$, CA9 and nestin are not significantly changed after RT and TMZ therapy. The VEGFR1 expression tends to be upregulated after the treatment. Original magnification, $\times 40$; scale bar, $100 \mu \mathrm{m}$. RT, radiotherapy; TMZ, temozolomide. 
A

B
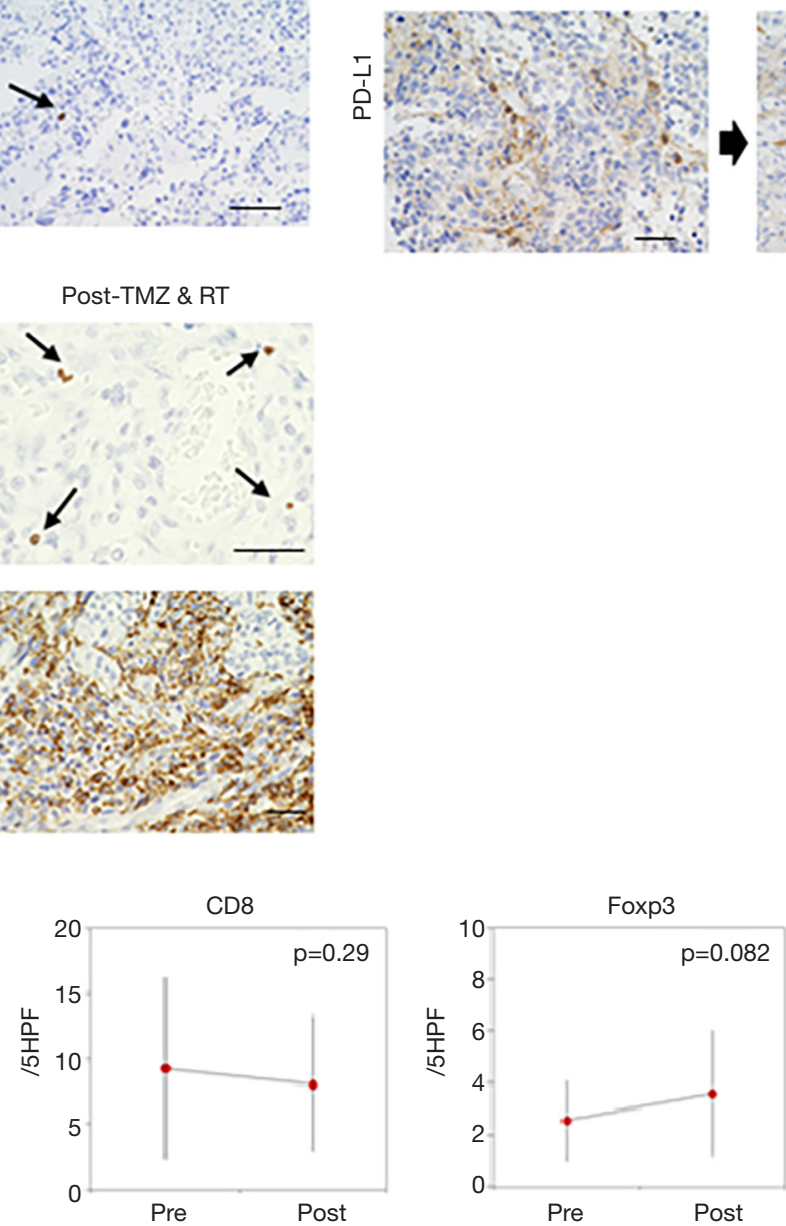

Post-TMZ \& RT
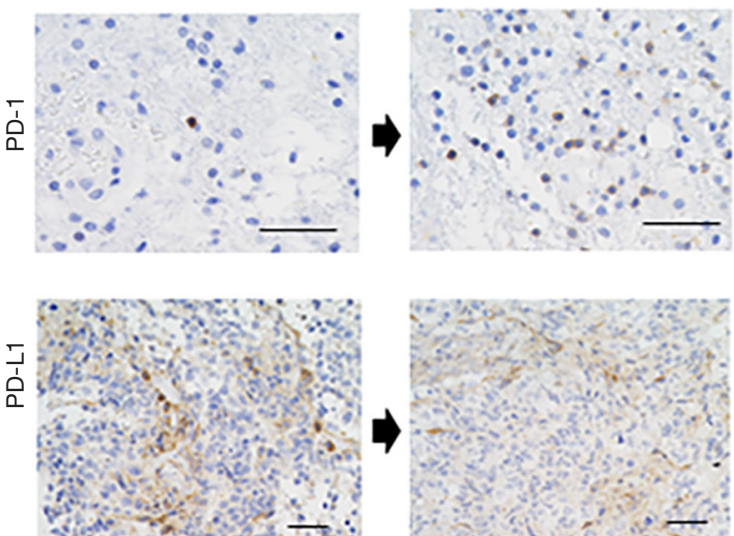
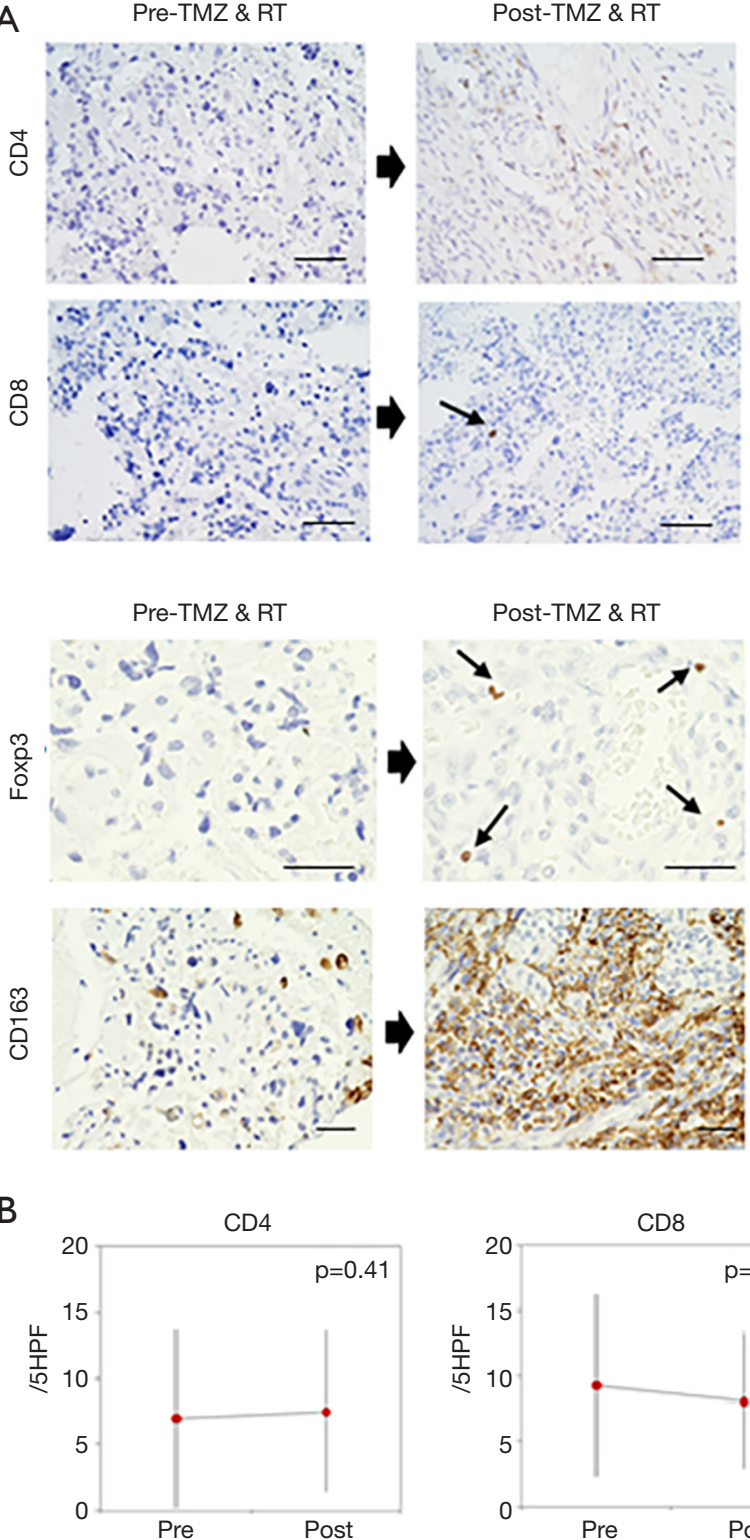

Post-TMZ \& RT
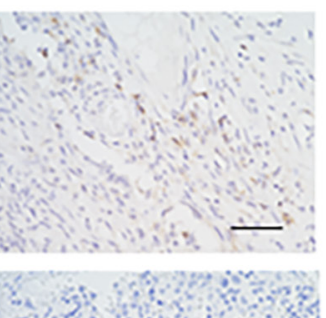

Post-TMZ \& RT
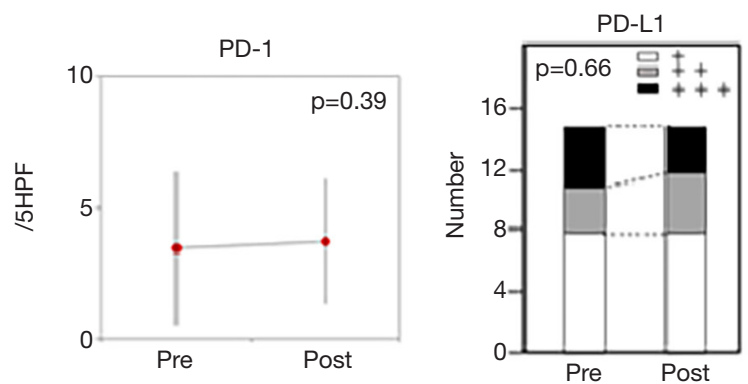

Figure 3 Immunohistochemical analyses of the tumor immune microenvironment. Photomicrographs (A) and the results of statistical analyses (B) are shown. Immunohistochemical analyses demonstrate that the expression levels of CD4, CD8, CD163, PD-1, and PD-L1 are not significantly changed after the treatment with RT and TMZ. The number of Foxp3 (+) cells tended to be increased after the treatment. Black arrow, CD8 (+) lymphocyte, and Foxp3 (+) cells. Original magnification, ×40; scale bar, $100 \mu \mathrm{m}$. RT, radiotherapy; TMZ, temozolomide. 

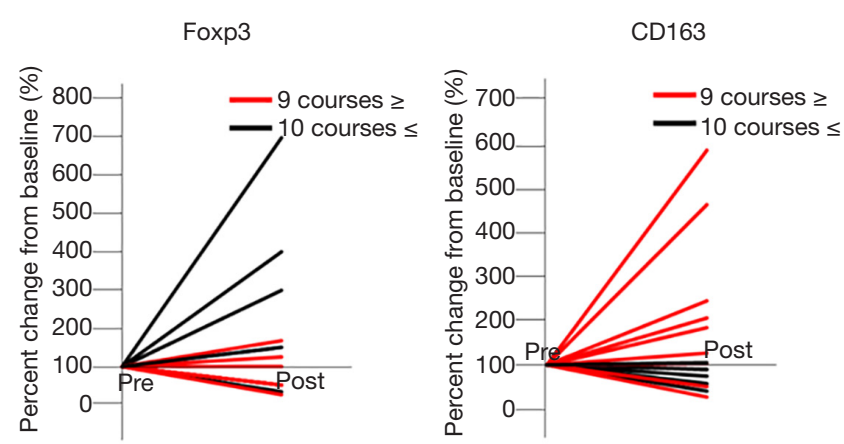

Figure 4 Percent change in the number of Foxp3 (+) cells and CD163 (+) cells. The percent change in the number of Foxp3 (+) cells and CD163 (+) cells from baseline (pre-RT and TMZ) is shown. The patients who received $\geq 10$ courses of TMZ exhibit a higher percent change in Foxp3 (+) cells and a lower percent change in CD163 $(+)$ cells in post-treatment tumors compared with that observed in patients who received $\leq 9$ courses of TMZ (Foxp3, $\mathrm{P}=0.058$; CD163, $\mathrm{P}=0.021$ ).

specimens obtained from patients with GB who underwent surgical resection in three different settings: GBs collected before any treatment (naïve GBs); GBs that were resected following Bev therapy (under the control of Bev); and recurrent GBs that were resected after long-term Bev therapy (Bev failure). In that study, Bev downregulated the expression of the PD-1/ PD-L1 immune checkpoint molecules, suppressed the infiltration of Tregs and TAMs, and increased CTL infiltration (5). The highlights of the changes in TME after the Bev administration (Bev failure) are shown using the original images in Figure 5.

\section{Discussion}

In the present study, the expression levels of CD34, VEGF-A, VEGFR2, HIF-1 $\alpha$, CA9, nestin, CD4, CD8, CD163, PD-1, and PD-L1 did not change significantly after RT and TMZ therapy (Table 1). The expression level of VEGFR1 and the number of Foxp3 (+) cells tended to be upregulated and increased after the treatment, respectively. Therefore, these results strongly support the hypothesis that Bev induces an immunosupportive microenvironment, as described previously (5).

Although alterations in the TME during chemotherapies, such as those that use carboplatin, cisplatin, and cyclophosphamide, have been previously reported, very few studies examined the changes in tumor angiogenesis and TME after treatment with RT and TMZ (2). The effect of
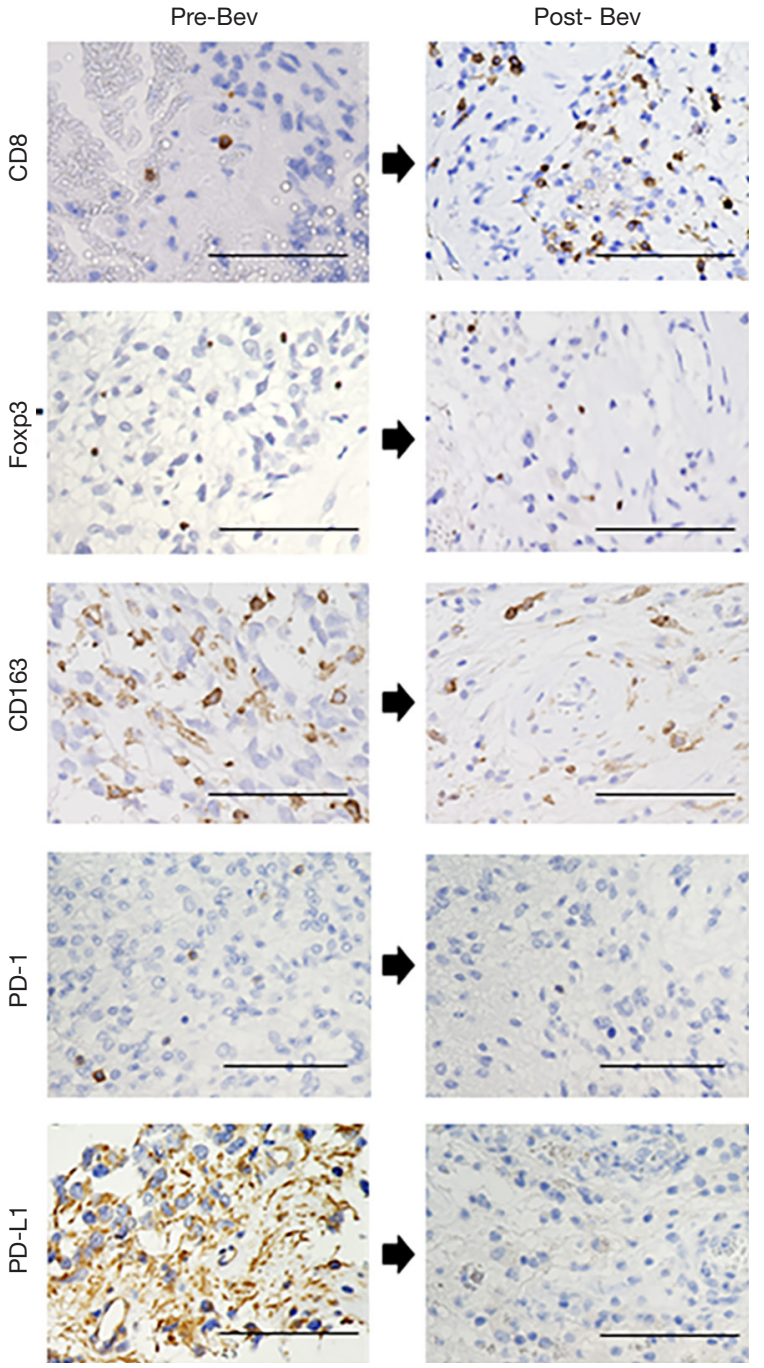

Figure 5 Highlights of the changes in the tumor-immune microenvironment after the administration of bevacizumab. Our previous study (9) reported the overall status of the TME after bevacizumab administration. Highlights of the changes in tumorimmune microenvironment are shown. Original magnification, $\times 40$; scale bar, $100 \mu \mathrm{m}$.

the RT and TMZ on tumor vascularity was supposed to be limited in animal models compared with that of Bev (10), whereas the continuous administration of low-dose TMZ (metronomic chemotherapy) reduced the MVD of recurrent GBs (11). In the present study, although MVD was not significantly increased after RT and TMZ, the expression of VEGFR1 tended to be upregulated, which might be associated with the abnormal vascular structure. A gradual deficit of pericyte is associated with blood-brain barrier 
(BBB) leakage, leading to severe vasogenic brain edema (12). The changes in vascular structure observed after RT and TMZ therapy must be evaluated using the present paired pre- and post-treatment samples.

Regarding immunomodulatory effects, RT and TMZ therapy might even worsen the immunosuppressive TME in GBs. A previous study demonstrated that the number of Tregs increased rapidly during long-term TMZ therapy in GBs, which is known as the rebound phenomenon (13). The present study also demonstrated that the patients who received $\geq 10$ courses of $T M Z$ showed a higher percent change in Foxp3 $(+)$ cells in post-treatment tumors compared with that observed in patients who received $\leq 9$ courses of TMZ. In contrast, our previous study revealed that the number of Foxp3 (+) cells was decreased when Bev was co-administered with RT and TMZ, despite the longterm administration of adjuvant TMZ (5). Furthermore, other studies showed that the number of Tregs was not increased when TMZ was co-administered with immunemodulatory agents, such as vaccination and interleukin-2 $(14,15)$. It may be difficult to understand the alteration of TME after the numerous combinational treatments. The expression of PD-1 was increased in recurrent GBs after RT and TMZ, which was associated with a shorter survival after recurrence (16). In the present study, the expressions of PD-1/PD-L1 were not upregulated in tumors after RT and TMZ compared with that observed in pre-treatment tumors. Further analysis is needed to confirm the changes in immune checkpoint molecules after the treatment.

Immunosuppressive TAMs in the peripheral blood were reported to be upregulated in the recurrent GBs after RT and TMZ (17). Interestingly, in the present study, the TAM status differed from the TMZ courses. Long-term administration of TMZ might sustain the status of TAMs, without their dramatic decrease, which was observed after Bev administration. Furthermore, macrophages express different functional programs in response to microenvironmental signals, which was termed M1/M2 polarization (18). Changes in M1 macrophages must be also examined to confirm the overall status of macrophages after RT and TMZ.

In contrast to TMZ, VEGF/VEGFR-targeted agents promote an immunosupportive TME (9). This finding was compatible with the results of our previous study (5). The previous study demonstrated that VEGF-A produced by the tumor enhances the expression of inhibitory immune checkpoints, thus mediating T-cell exhaustion on intratumoral CD8 (+) T cells. Therefore, a combinational approach using anti-VEGF-A and anti-PD-1 antibodies yielded synergistic effects on the CT26 mouse model of colorectal cancer (9). In particular, the status of Tregs exhibited dynamic changes after the treatment with VEGF/VEGFR-targeted agents. VEGF/VEGFR-targeted therapies, such as Bev, sorafenib, and sunitinib, reduce the population of Tregs in the peripheral blood and tumor tissues in GBs, metastatic colorectal cancer, hepatocellular carcinoma, and renal cell carcinoma $(19,20)$. However, the status of the TME is highly controversial in the recurrent stage, after anti-angiogenic therapy. Tregs are increased in the peripheral blood of patients with recurrent GBs after resistance to VEGFR inhibitors (21). The upregulation of PD-1 on CD4 (+) and CD8 (+) T cells was also reported in patients with GBs or metastatic renal cell carcinoma refractory to VEGFR-targeted therapy (21). The reason why the status of TME in the recurrent stage after antiangiogenic therapy is controversial, which may be caused by the difference of analyzed samples between systemic circulation (peripheral blood) and local lesion (tumor specimens), in addition to the difference in the target of inhibitors. Tada et al. suggested that analyses of lymphocytes and other immune cells using tumor specimens are more important than analyses of peripheral blood for the investigation of cancer immunology (22). Although most previous studies used peripheral blood mononuclear cells, studies using tumor specimens obtained surgically before and after treatment are considered to be a powerful tool to investigate the biological effects of targeted therapies. Comparative analyses of tumor samples and peripheral blood may be required for monitoring immunosuppressive cells and molecules. Furthermore, the response rate of targeted therapies also contributes to changes of the TME. For example, Tregs, PD-L1 and TAMs are upregulated in patients with metastatic renal cell carcinoma who exhibit a partial response (23). Further investigations are required to determine the differences in therapeutic actions based on the response, dosage and duration of TMZ therapy.

A limitation of the present study was the paucity of the number of paired tissues from the same patients due to the relative rarity of salvage surgery for recurrent GBs following failure of the RT and TMZ. Studies using a larger number of patients are warranted to confirm and generalize the findings of the present study.

\section{Conclusions}

This was the first study to show the alterations of the TME following RT and TMZ therapy using paired pre- and post- 
treatment samples. The RT and TMZ standard therapy did not yield an immunosupportive effect in patients with malignant glioma.

\section{Acknowledgments}

The authors greatly thank Ms. Naoko Tsuzuki at the Department of Neurosurgery, Keio University School of Medicine and Ms. Eri Honzawa at the Division of Diagnostic Pathology, Jikei University School of Medicine Kashiwa Hospital, for technical assistance with laboratory work and sample preparation.

Funding: This study was supported by the Ministry of Education, Culture, Sports, Science and Technology and the Japan Society for the Promotion of Science (KAKENHI), Grant/Award Number: 18J21382.

\section{Footnote}

Conflicts of Interest: TT serves as an unpaid editorial board member of Annals of Translational Medicine from Dec 2019 to Nov 2021. The other authors have no conflicts of interest to declare.

Ethical Statement: The authors are accountable for all aspects of the work in ensuring that questions related to the accuracy or integrity of any part of the work are appropriately investigated and resolved. All procedures in the present study were in accordance with the ethical standards of the institutional and/or national research committee and with the 1964 Helsinki declaration and its later amendments or comparable ethical standards. This study was approved by the Institutional Review Board of Keio University School of Medicine and Jikei University Kashiwa Hospital (Reference number: 20050002 and 26334). Written consent was obtained from all patients.

Open Access Statement: This is an Open Access article distributed in accordance with the Creative Commons Attribution-NonCommercial-NoDerivs 4.0 International License (CC BY-NC-ND 4.0), which permits the noncommercial replication and distribution of the article with the strict proviso that no changes or edits are made and the original work is properly cited (including links to both the formal publication through the relevant DOI and the license). See: https://creativecommons.org/licenses/by-nc-nd/4.0/.
1. Polyak K, Haviv I, Campbell IG. Co-evolution of tumor cells and their microenvironment. Trends Genet 2009;25:30-8.

2. Tamura R, Tanaka T, Akasaki Y, et al. The role of vascular endothelial growth factor in the hypoxic and immunosuppressive tumor microenvironment: perspectives for therapeutic implications. Med Oncol 2019;37:2.

3. Gabrilovich DI, Ostrand-Rosenberg S, Bronte V. Coordinated regulation of myeloid cells by tumours. Nat Rev Immunol 2012;12:253-68.

4. Ohm JE, Gabrilovich DI, Sempowski GD, et al. VEGF inhibits T-cell development and may contribute to tumorinduced immune suppression. Blood 2003;101:4878-86.

5. Tamura R, Tanaka T, Ohara K, et al. Persistent restoration to the immunosupportive tumor microenvironment in glioblastoma by bevacizumab. Cancer Sci. 2019;110:499-508.

6. Stupp R, Mason WP, van den Bent MJ, et al. Radiotherapy plus concomitant and adjuvant temozolomide for glioblastoma. N Engl J Med. 2005;352:987-96.

7. Tamura R, Tanaka T, Miyake K. Histopathological investigation of glioblastomas resected under bevacizumab treatment. Oncotarget. 2016;7:52423-35.

8. Yamamoto Y, Tamura R, Tanaka T, et al. "Paradoxical" findings of tumor vascularity and oxygenation in recurrent glioblastomas refractory to bevacizumab. Oncotarget 2017;8:103890-9.

9. Voron T, Colussi O, Marcheteau E, et al. VEGF-A modulates expression of inhibitory checkpoints on CD8+ T cells in tumors. J Exp Med 2015;212:139-48.

10. Grossman R, Brastianos H, Blakeley JO, et al. Combination of anti-VEGF therapy and temozolomide in two experimental human glioma models. J Neurooncol 2014;116;59-65.

11. Woo JY, Yang SH, Lee YS, et al. Continuous low-dose temozolomide chemotherapy and microvessel density in recurrent glioblastoma. J Korean Neurosurg Soc 2015;58;426-31.

12. Dubois LG, Campanati L, Righy C. Gliomas and the vascular fragility of the blood brain barrier. Front Cell Neurosci 2014;12;8:418.

13. Batich KA, Reap EA, Archer GE, et al. Long-term Survival in Glioblastoma with Cytomegalovirus pp65-Targeted Vaccination. Clin Cancer Res. 2017;23:1898-909.

14. Mitchell DA, Cui X, Schmittling RJ, et al. Monoclonal antibody blockade of IL-2 receptor during lymphopenia selectively depletes regulatory $\mathrm{T}$ cells in mice and humans.

\section{References}


Blood. 2011;118:3003-12.

15. Sampson JH, Schmittling RJ, Archer GE, et al. A pilot study of IL-2R $\alpha$ blockade during lymphopenia depletes regulatory T-cells and correlates with enhanced immunity in patients with glioblastoma. PLoS One 2012;7:e31046.

16. Miyazaki T, Ishikawa E, Matsuda M, et al. Assessment of PD-1 positive cells on initial and secondary resected tumor specimens of newly diagnosed glioblastoma and its implications on patient outcome. J Neurooncol. 2017;133:277-85.

17. Lu-Emerson C, Snuderl M, Kirkpatrick ND, et al. Increase in tumor-associated macrophages after antiangiogenic therapy is associated with poor survival among patients with recurrent glioblastoma. Neuro Oncol 2013;15;1079-87.

18. Tamura R, Tanaka T, Yamamoto Y, et al. Dual role of macrophage in tumor immunity. Immunotherapy 2018;10:899-909.

19. Adotevi O, Pere H, Ravel P, et al. A decrease of regulatory $\mathrm{T}$ cells correlates with overall survival after sunitinib-based

Cite this article as: Tamura $\mathrm{R}$, Tanaka $\mathrm{T}$, Morimoto $\mathrm{Y}$, Kuranari Y, Yamamoto Y, Takei J, Murayama Y, Yoshida K, Sasaki H. Alterations of the tumor microenvironment in glioblastoma following radiation and temozolomide with or without bevacizumab. Ann Transl Med 2020;8(6):297. doi: 10.21037/atm.2020.03.11 antiangiogenic therapy in metastatic renal cancer patients. J Immunother 2010;33:991-8.

20. Desar IME, Jacobs JH, Hulsbergen-vandeKaa CA, et al. Sorafenib reduces the percentage of tumour infiltrating regulatory $\mathrm{T}$ cells in renal cell carcinoma patients. Int $\mathrm{J}$ Cancer 2011;129:507-12.

21. Du Four S, Maenhout SK, Benteyn D, et al. Disease progression in recurrent glioblastoma patients treated with the VEGFR inhibitor axitinib is associated with increased regulatory $\mathrm{T}$ cell numbers and $\mathrm{T}$ cell exhaustion. Cancer Immunol Immunother 2016;65:727-40.

22. Tada $Y$, Togashi $Y$, Kotani D, et al. Targeting VEGFR2 with Ramucirumab strongly impacts effector/ activated regulatory $\mathrm{T}$ cells and CD8+ $\mathrm{T}$ cells in the tumor microenvironment. J Immunother Cancer 2018;6:106.

23. Wallin JJ, Bendell JC, Funke R, et al. Atezolizumab in combination with bevacizumab enhances antigen-specific T-cell migration in metastatic renal cell carcinoma. Nat Commun 2016;7:12624. 